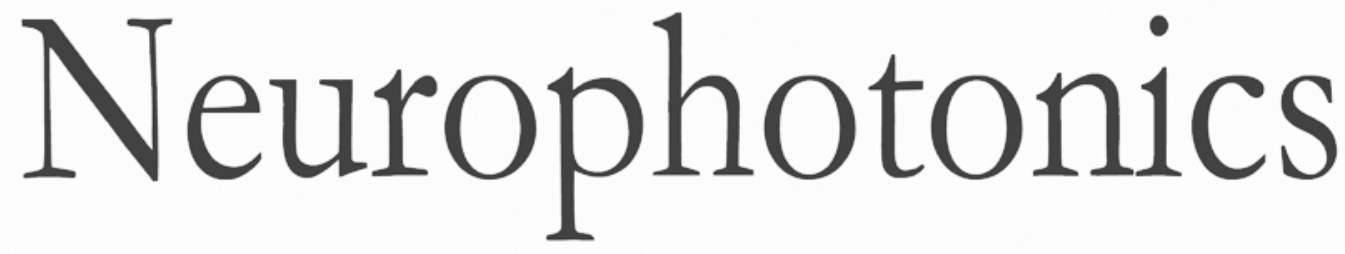

\title{
Quantitative electroencephalograms and neuro-optometry: a case study that explores changes in electrophysiology while wearing therapeutic eyeglasses
}

Deborah Zelinsky

Corey Feinberg 


\title{
Quantitative electroencephalograms and neuro-optometry: a case study that explores changes in electrophysiology while wearing therapeutic eyeglasses
}

\author{
Deborah Zelinsky ${ }^{\mathrm{a}, \star}$ and Corey Feinberg ${ }^{\mathrm{b}}$ \\ ${ }^{a}$ The Mind-Eye Connection, Northbrook, Illinois, United States \\ ${ }^{b}$ Meridian Behavioral Health Services, Northbrook, Illinois, United States
}

\begin{abstract}
The brain is equipped with a complex system for processing sensory information, including retinal circuitry comprising part of the central nervous system. Retinal stimulation can influence brain function via customized eyeglasses at both subcortical and cortical levels. We investigated cortical effects from wearing therapeutic eyeglasses, hypothesizing that they can create measureable changes in electroencephalogram (EEG) tracings. A Z-Bell ${ }^{\mathrm{SM}}$ test was performed on a participant to select optimal lenses. An EEG measurement was recorded before and after the participant wore the eyeglasses. Equivalent quantitative electroencephalography (QEEG) analyses (statistical analysis on raw EEG recordings) were performed and compared with baseline findings. With glasses on, the participant's readings were found to be closer to the normed database. The original

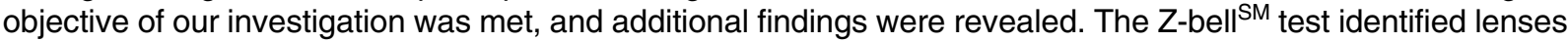
to influence neurotypical brain activity, supporting the paradigm that eyeglasses can be utilized as a therapeutic intervention. Also, EEG analysis demonstrated that encephalographic techniques can be used to identify channels through which neuro-optomertric treatments work. This case study's preliminary exploration illustrates the potential role of QEEG analysis and EEG-derived brain imaging in neuro-optometric research endeavors to affect brain function. @ 2017 Society of Photo-Optical Instrumentation Engineers (SPIE) [DOI: 10.1117/1.NPh.4.1.011013]
\end{abstract}

Keywords: neurophotonics; brain mapping; visual system; sensory disorders; signal processing; neuro-optometry.

Paper 16074SSRR received Dec. 20, 2016; accepted for publication Feb. 24, 2017; published online Mar. 15, 2017.

\section{Introduction}

The 126 million photosensitive cells in each retina are formed from brain tissue and are considered part of the central nervous system. Using customized eyeglasses that disperse light onto the complex retinal circuitry is an example of neurophotonics-an emerging discipline in science dealing with optical methods that affect neurological systems. This case study investigated the retina's point-to-point correspondence with cortical locations to determine whether customized eyeglasses altered brain activity and sensory processing. Although only one case is presented in this article, we have seen similar effects of the eyeglasses on the EEG findings in other clinical cases (data not shown).

Visual stimuli trigger specific neurons to emit electrical impulses. The idea of stimulating the retina to selectively alter neural pathways is supported by the discovery in 2002 of several types of intrinsically photosensitive retinal ganglion (ipRGC) cells, ${ }^{1}$ which comprise part of the retinohypothalamic tract and react to changes in external luminance. The combination of those external luminance signals, with internal signals from the habenula and intergeniculate leaflet, provide quantifiable feedback about a patient's physiological state. ${ }^{2}$ In other words, neurochemistry is theoretically affected by retinal stimulation.

*Address all correspondence to: Deborah Zelinsky, E-mail: mindeyeconnection@ msn.com
As shown in Fig. 1, there are many retinal projections traveling through more pathways than simply eyesight. Some are linked with neurological systems, such as the superior colliculus (retinotectal pathway) (Sauve and Gaillard 1995), ${ }^{3}$ and others are linked with chemical structures, such as the superior chiasmatic nucleus of the hypothalamus (retinohypothalamic pathway) ${ }^{4}$ or the dorsal raphe nucleus (retinoraphe pathway). ${ }^{5}$ Many of those connections are bidirectional and beneath conscious processing levels.

\section{Materials and Methods}

One method of stimulating the retina involves nontraditional eyeglasses intended to scatter light on peripheral areas of the retina, rather than on classic central eyesight areas. ${ }^{6,7}$ Glasses can be designed to filter and disperse light in various ways, triggering signaling changes in a multitude of retinal pathways. This stimulation influences the two-way processing in both cortical eyesight and subcortical neural pathways.

Quantitative analysis offers valuable information about patterns of cortical activation. Clusters of synchronized neural activity create outputs that can be recorded and measured in electroencephalograms (EEG). Quantitative electroencephalography (QEEG) is the process of taking raw EEG recordings and performing statistical analysis. ${ }^{8}$ This often involves comparing findings for individual patients with the population norms. Similarly, brain mapping using EEG recordings can measure

$2329-423 X / 2017 / \$ 25.00$ (C) 2017 SPIE 


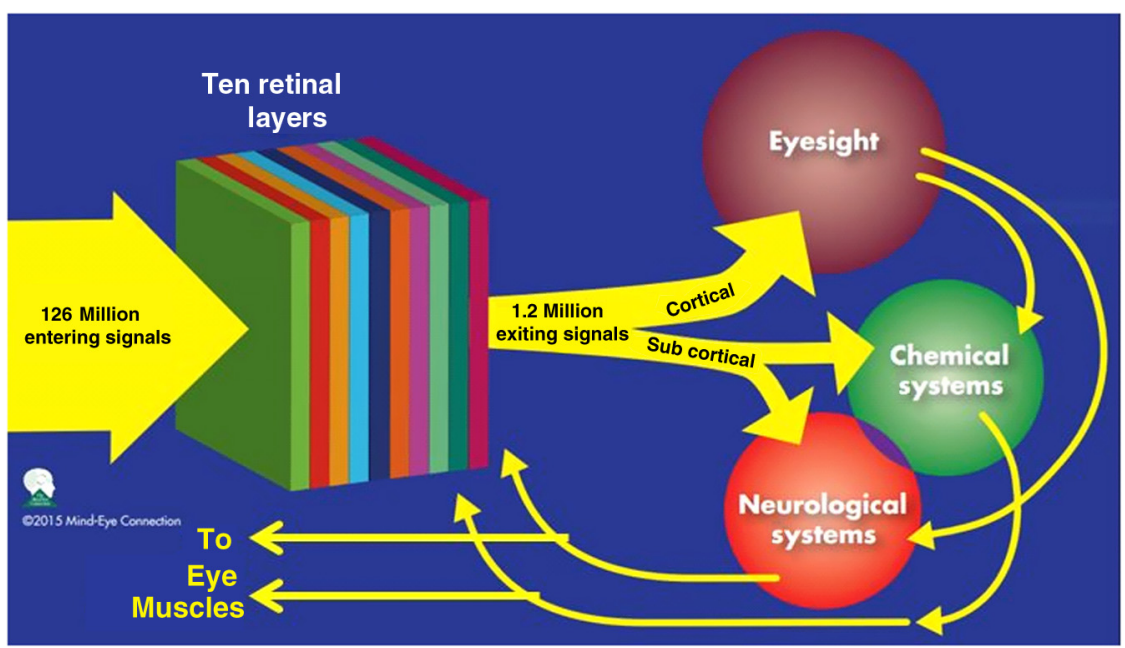

Fig. 1 There are many subcortical neurological and chemical pathways that create complexity in the various visual systems. Most of the exiting signals through the optic nerve are used for cortical eyesight. However, eyesight signals are slower than the subcortical ones from the brainstem and limbic system. Reprinted with permission from the Mind-Eye Connection.

the effects of customized eyeglasses on brain activity, allowing investigation of cortical effects from wearing therapeutic eyeglasses. In this case example, statistical measures were conducted for both surface values (outer cortex) and subcortical current source densities using low-resolution electromagnetic tomography (LORETA) analysis techniques to confirm the EEG findings. ${ }^{9}$

In this case, an IRB board was not utilized. It should be noted that this was not a controlled experiment due to multiple factors. One of these factors was the clinical setting where the experiment was conducted. For instance, there was no standardization of lighting, chair position, or patient head position. Additionally, the participant did not go through a screening process, and no formal inclusion criteria were established. For these reasons, this exploratory investigation should be considered as a clinical case example and would not pass the scrutiny of an IRB review. It should be recognized that the findings of this investigation are limited to that extent and should serve as a foundation for future controlled research projects.

An 18-year-old participant volunteered for this experiment. An informed consent was obtained from him and his father. The hypothesis was that his EEG findings would be changed by wearing eyeglasses. A classic visual examination was performed, including eyesight, eye aiming, and focusing tests, along with a Z-Bell ${ }^{\mathrm{SM}}$ test to evaluate the interaction between auditory and visual localization abilities. The patient had his eyes closed and was instructed to point to the exact location of a ringing bell. Mind-Eye protocol was followed to determine the specific type of lens, which is necessary to have the patient succeed at finding the bell location. The Mind-Eye protocol included obtaining an optimal lens prescription for the peripheral retina by testing with both eyes open simultaneously and emphasizing patient comfort and sensory integration. This is a different prescription from an optimal lens for 20/20 central acuity, which is typically obtained by monocular testing for clarity. All test results were used to prescribe a set of lenses addressing the patient's ability to solidly integrate visual and auditory signals. This type of lens was not for central acuity but for peripheral retinal stimulation. The lens was used to induce a balance between peripheral and central retinal stimulation as well as between cortical and subcortical activity.

More specifically, in this case, the participant's custom lenses were designed to direct the light to the lower portion of his retina. This location was the pathway that allowed him to touch the bell most accurately. His customized lenses were thicker on the bottom edges than the top edges, bending the entering light toward the base of an induced prism and diverting the light downward on the lower retina of each eye. Lehman et al. ${ }^{10}$ demonstrated a latency difference in the visual cortex between stimulation of the superior retina as compared to the inferior retina. Even though the signals from each location reached the visual cortex at the same time, the research concluded that the cortex reacted faster (lower latency) to superior retinal stimulation than to stimulation of the inferior retina. The lower retinas feed information through the temporal lobes on the way to the visual cortex, as opposed to the superior retina, which transmits signals through the parietal lobes.

EEG tracings were recorded for the volunteer, with and without customized eyeglasses comparing results with population norms using the "Thatcher Lifespan Reference Database." While the participant's eyes were closed, a baseline EEG was recorded for $5 \mathrm{~min}$ at 19 locations across the patient's scalp, without the eyeglasses. The baseline EEG analysis had findings that deviated from normal readings primarily in temporal and occipital brain regions. A second EEG was recorded after customized lenses were placed in front of the participant's eyes. Equivalent QEEG analyses for surface values as well as current source density were performed and compared with baseline findings. The analysis with glasses found a statistically significant shift in brain function.

\section{Results}

Measurable changes were found in brain EEG tracings, depending on the eyeglass lenses worn, suggesting that specific eyeglasses can be used as a noninvasive manner of intentionally altering brain activity.

The QEEG provides information about how an individual's electrophysiology as compared to age-specific and gender norm 


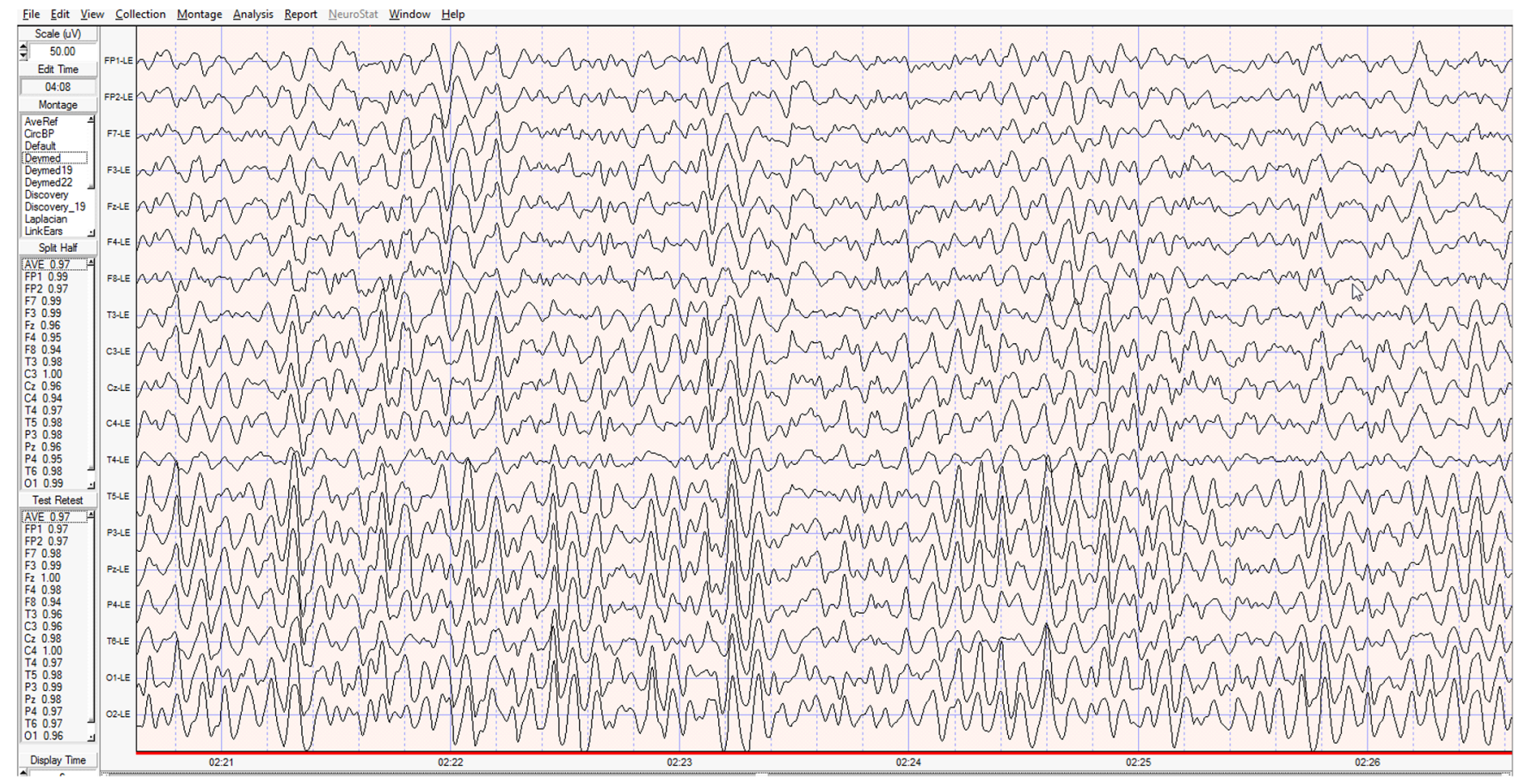

Fig. 2 EEG before customized lenses.

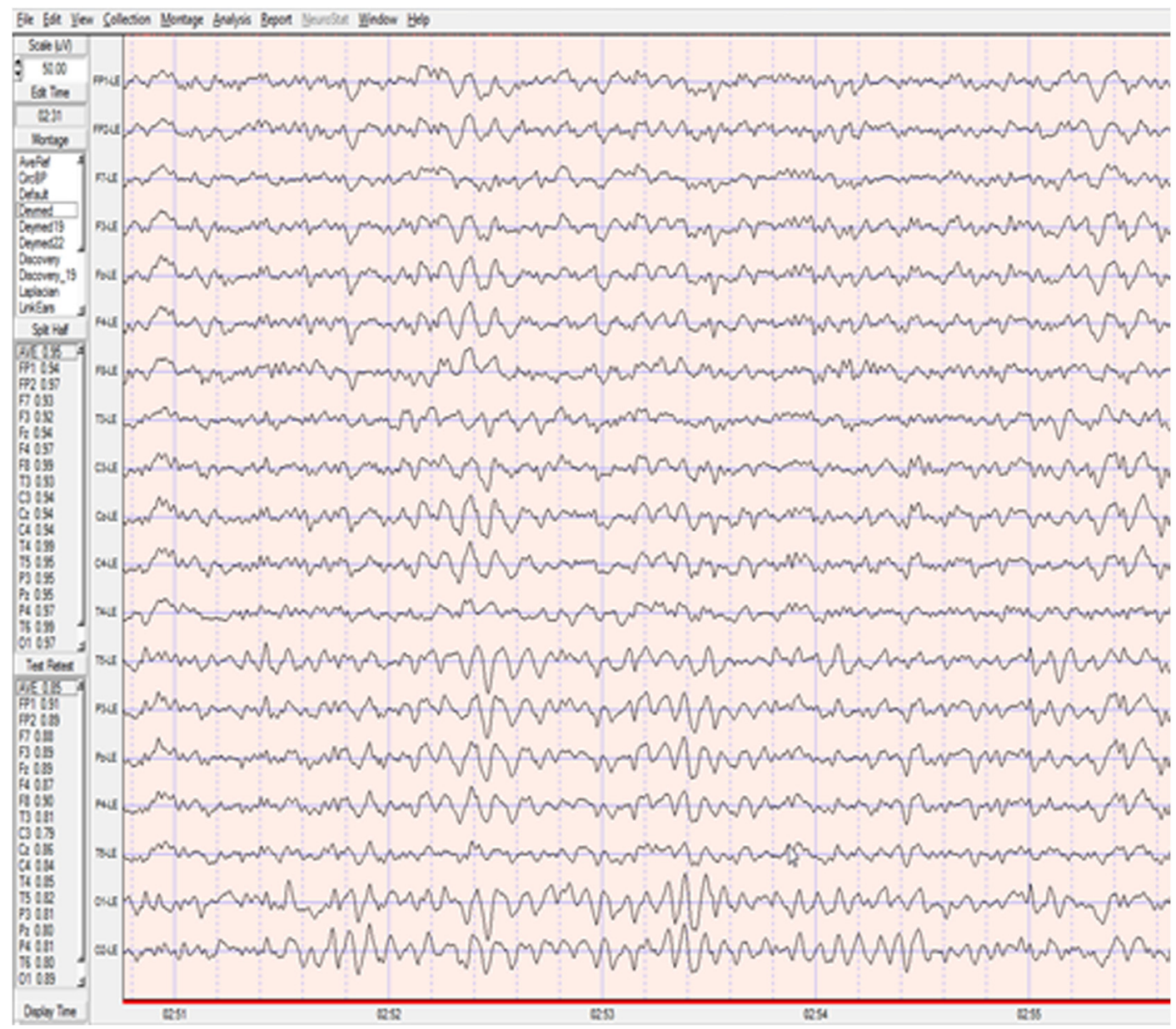

Fig. 3 EEG immediately after customized eyeglasses were worn by the participant. 
values. Participant's baseline EEG analysis revealed deviations from the database in power (amount of energy) primarily in the temporal and occipital regions of the brain. While the participant wore the customized glasses, QEEG readings were found to be more neurotypical when compared with the normed database. Customized glasses reduced the degree of deviation from the norm.

An EEG was recorded with the participant's eyes closed for a minimum of $300 \mathrm{~s}$; the EEG was edited for artifact and analyzed for coherence, phase, amplitude differences, and relative power. These measures were then used for comparison with the Thatcher Lifespan Reference Database using the appropriate age-matched group. Deviations from the database are expressed in Z-scores, which indicate the relationship to the population norm for a given metric. The participant's baseline EEG analysis revealed Z-scores representing increases in power (amount of energy) exceeding $+3.09 \mathrm{SD}$ and were statistically significant to the $p<0.001$ level of probability. When the participant wore the glasses, EEG recordings showed significant reductions in Z-scored deviations indicating greater similarity to their neurotypicaldat equivalents in the normed database.

Figure 2 shows how there were some abnormalities in EEG waves before the use of the customized lenses. Figure 3 shows normalization of the EEG waves and the more neurotypical QEEG.

\section{Z Scored FFT Absolute Power}
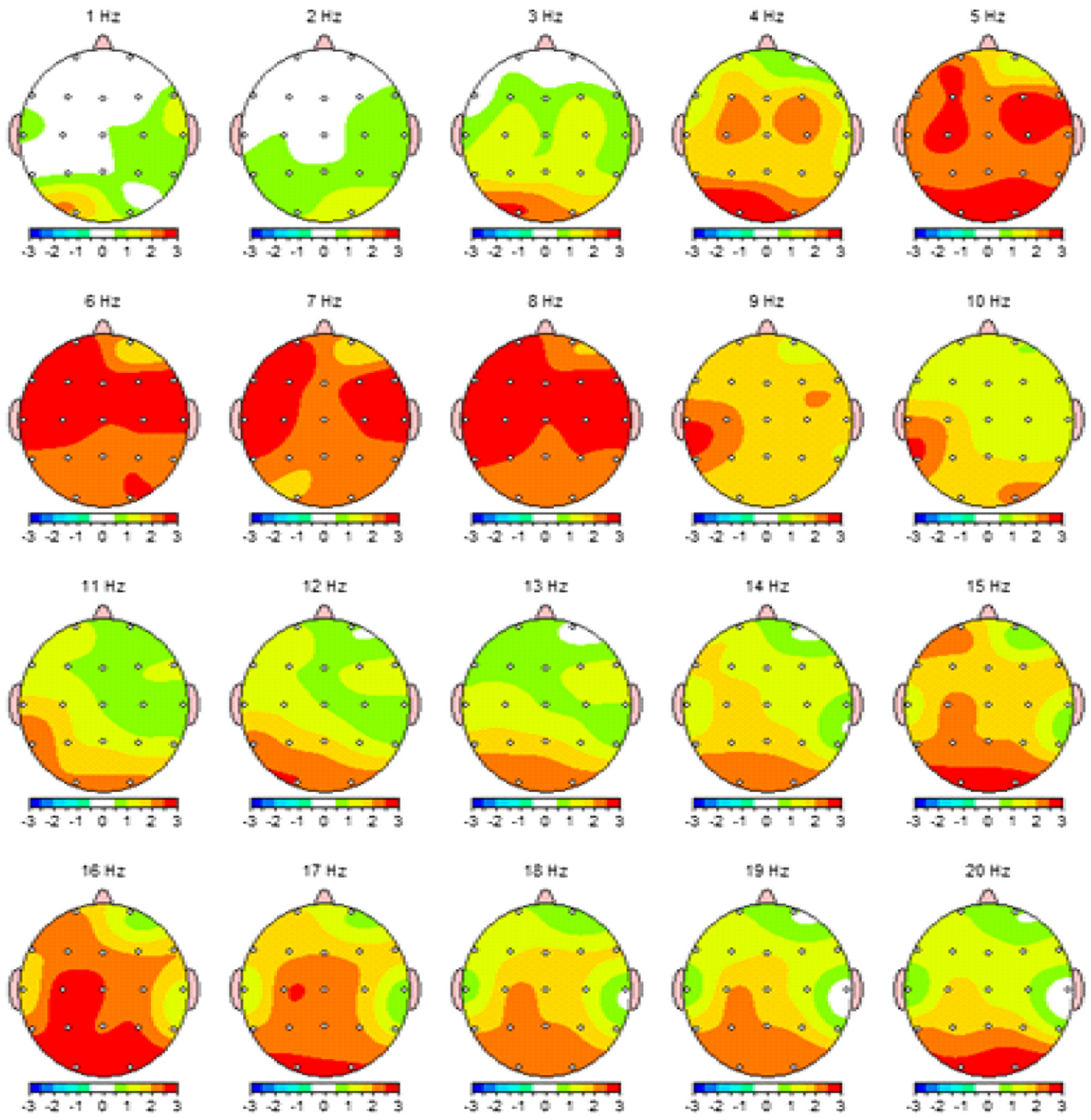

Fig. 4 QEEG before customized eyeglasses. 


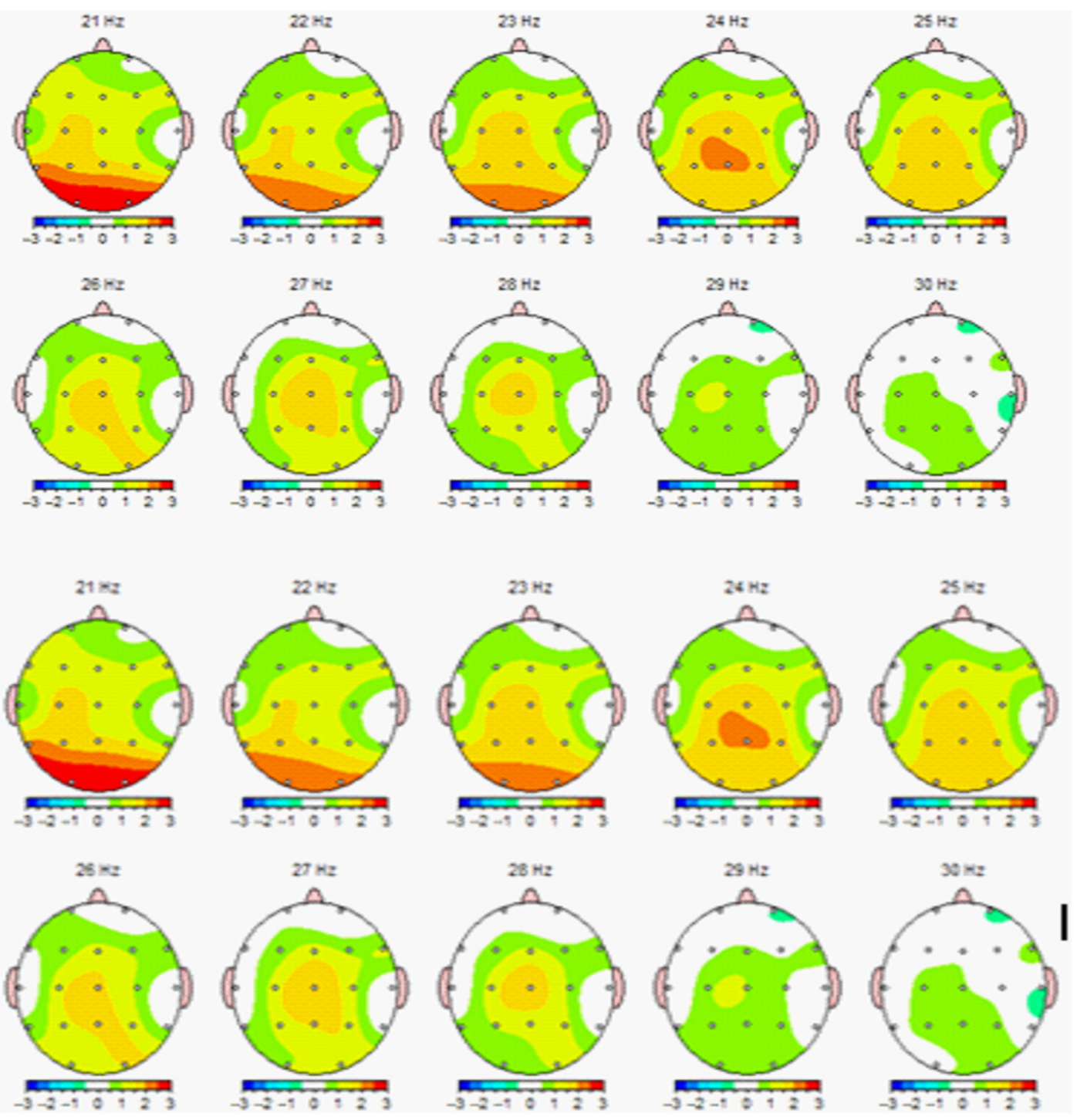

Fig. 5 QEEG immediately after eyeglasses were placed on the participant.

Figures 4-7 show the immediate changes noted by the use of customized eyeglasses that had been selected for the individual patient. The lenses were selected based on optometric testing, which included a Z-Bell ${ }^{\mathrm{SM}}$ test (described above). When the patient was wearing those lenses that best synchronized their auditory and visual localization abilities, electrical activity in the brain was more neurotypical.

\section{Discussion}

This single patient case study had an abnormality in the baseline testing. We thought that the presence of baseline abnormality was relevant, but the specific pattern of abnormality of this individual was not. His EEG findings were altered by the therapeutic eyeglasses. It seemed more sensible to use a volunteer patient with an abnormality to determine if the lens effect moved the

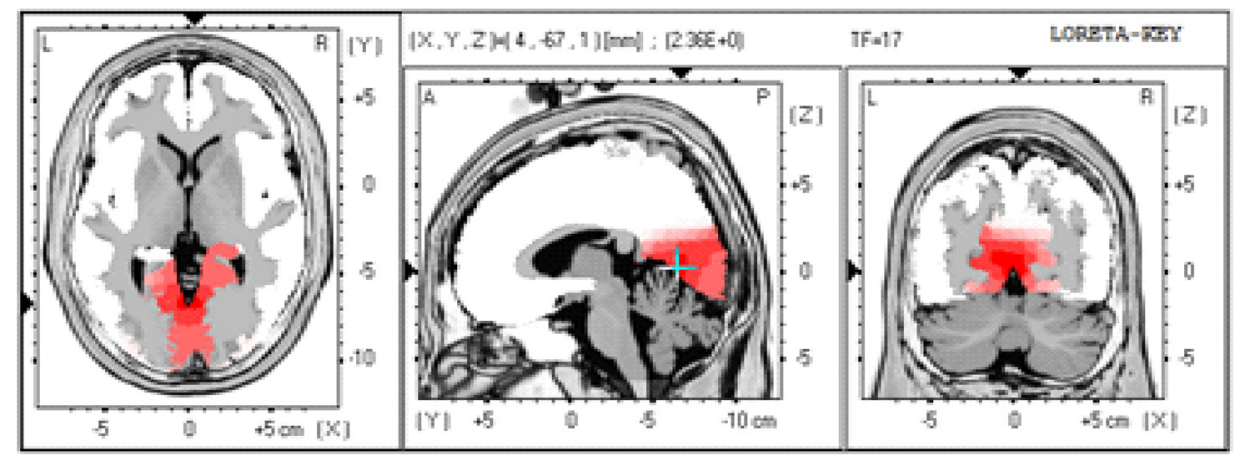

Fig. 6 LORETA indicating spots that were more than three standard deviations away from the norm. 


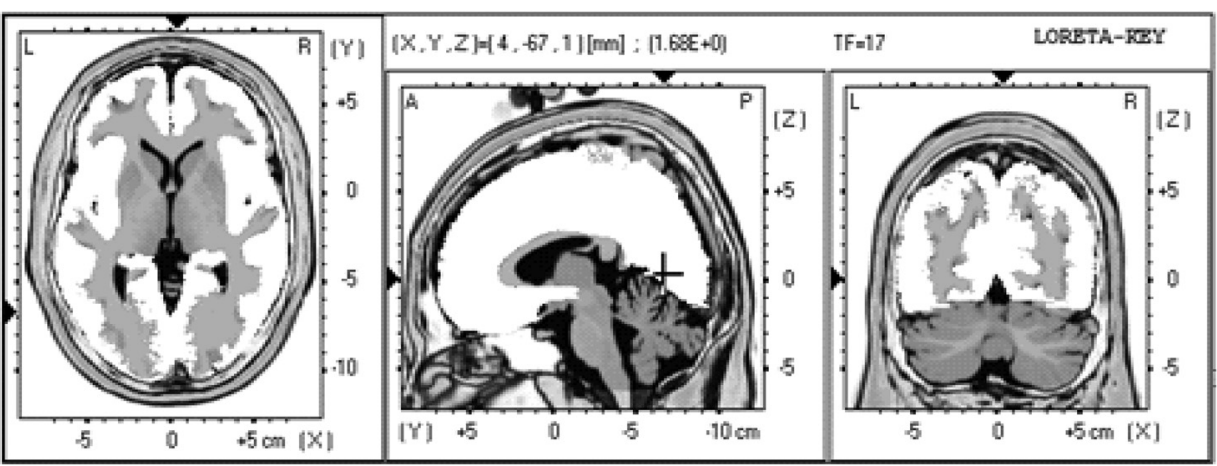

Fig. 7 LORETA immediately after lenses were placed on the volunteer.

Z-scores toward normalization, thus suggesting the possibility of a therapeutic usage for eyeglasses. If we had used a volunteer with normal baseline findings, we could have demonstrated an effect, but not necessarily whether the effect moved toward normalization.

Each eye has $\sim 126$ million light-sensitive receptors120 million rods and 6 million cones in the retinal photoreceptor layer-and roughly 12 thousand (1\% of 1.2 million cells) in the retinal ganglion cell layer. As shown in Fig. 1, those 126 million inputs travel through a sophisticated filtering system (containing its own circadian clock ${ }^{11-13}$ and immune system ${ }^{14,15}$ ) resulting in 1.2 million signals traveling in the optic nerve and optic radiations. ${ }^{16}$ Among the multitude of retinal connections, there are electrical and chemical circuitry pathways that are involved in retinal functions, but not necessarily eyesight. Some of the signals exit the eye via retinofugal fibers. Others return to the retina, via retinopetal fibers. The exiting signals first stimulate subcortical areas involving the brainstem, cerebellum, and limbic system, followed by slower signals traveling in cortical areas. Changes in visual signaling traveling through the pointto-point retinotopic brain mapping are quantifiable using modern technology. ${ }^{17}$ Thus, use of customized lenses as a therapeutic instrument in a clinical setting is an innovative way to alter brain mapping using the underlying concepts of neurophotonics.

In this participant's customized eyeglasses, light was directed onto the inferior retina. Signals that travel from the inferior portion of the retina are routed through the temporal lobes on their way to the visual cortex. Thus, using glasses to stimulate the inferior retina would be hypothesized to alter brain activity in the temporal lobes. This temporal lobe activation was verified during the analysis when comparing the activity with and without the lenses.

Customized eyeglasses act as an environmental filter. The incoming light has the ability to influence and direct the manner in which the eyes receive visual information from the external environment. Manipulating visual sensory input can alter the biochemical and neurological visual processing pathways in the brain. ${ }^{18}$ The expression of these different pathways should produce different patterns of electrophysiology, measurable by the EEG. Therefore, the hypothesis is that wearing customized eyeglasses can create measurable changes in EEG tracings. ${ }^{19,20}$

QEEG testing is highly reliable. Without interventions, results will remain consistent. ${ }^{21}$ In this single case, therapeutic eyeglasses were the sole intervention, inducing an effect within seconds of being worn. LORETA uses algorithms to predict current source densities, or source correlations, from the surface values. Values from LORETA have been validated by both
MRI and fMRI studies. The high test-retest reliability of QEEG measurements and the validation from LORETA testing suggest that the normalized effect on brain activity was due to the eyeglasses.

Objective testing provided evidence that prescribed customized lenses influenced brain activity. The reduction in power deviations from the database suggest that the participant's brain became more neurotypical when the participant wore the customized glasses. Finally, the QEEG analysis demonstrated that modern encephalographic techniques might be used to identify the channels through which neuro-optomertric treatments work. Although the initial hypothesis is supported by this case study, the need for more research is evident.

The concept of using eyeglasses to alter brain activity was supported by reliable measurements of more neurotypical electrical activity when the glasses were worn. By comparing and contrasting the volunteer's QEEG results with normed group data (before and after use of therapeutic eyeglasses), the $\mathrm{Z}$-Bell ${ }^{\mathrm{SM}}$ test results showed the benefits of prescribing customized lenses. The effectiveness of $\mathrm{Z}$-bell ${ }^{\mathrm{SM}}$ testing has far reaching potential uses, such as possibly delineating subtle differences between postconcussive syndrome and posttraumatic stress disorder. ${ }^{22,23}$ Influencing brain function through subcortical and cortical pathways other than those used for eyesight warrants further research.

The original aim of this study was to investigate if changes in EEG rhythms could be observed when customized eyeglasses were worn. The theory was based on neurophotonics-that light affects neurological systems and that the retina is a part of the central nervous system. This case study demonstrated the concept of prescribed eyeglasses influencing the brain toward a state of normalization, and additional findings were revealed.

\section{Conclusion}

The results of this exploration offer support to the paradigm that customized eyeglasses, in addition to other neuro-optometric treatments, can be utilized as a therapeutic intervention. The usage of therapeutic lenses to alter sensory signaling can be an adjunct to many types of practitioners during rehabilitation, providing the potential to decrease the required number of therapy sessions, thus saving time and money. Neuro-optometric lenses may broaden available treatment avenues and offer new hope for patients who have autonomic nervous system problems, neurodegenerative conditions, or injuries affecting motor skills and/or perception. Additionally, this preliminary exploration may serve to better understand the potential role 
of QEEG analysis and EEG-derived brain imaging in neurooptometric research endeavors.

\section{Disclosures}

Neither author has any conflict of interest.

\section{Acknowledgments}

Albert A. Sutton, O.D., discussed the retina as being part of the brain for decades of his long illustrious career in optometry. It is nice to see that his hard work is finally being documented by research.

\section{References}

1. S. Hattar et al., "Melanopsin-containing retinal ganglion cells: architecture, projections, and intrinsic photosensitivity," Science 295(5557), 1065-1070 (2002).

2. V. Fimreite, K. J. Ciuffreda, and N. K. Yadav, "Effect of luminance on the visually-evoked potential in visually-normal individuals and in mTBI/concussion," Brain Inj. 29(10), 1199-1210 (2015).

3. Y. Sauve and F. Gaillard, "The Organization of the Retina and Visual System," Chapter 33 in Regeneration in the Visual System of Adult Mammals, H. Kolb, E. Fernandez, and R. Nelson, Ed., University of Utah Health Sciences Center, Salt Lake City, Utah (1995).

4. L. P. Morin, "A path to sleep is through the eye (1, 2, 3)," eNeuro 2(2), 1-18 (2015).

5. T. Zhang et al., "ON and OFF retinal ganglion cells differentially regulate serotonergic and GABAergic activity in the dorsal raphe nucleus," Sci. Rep. 6, 26060 (2016).

6. J. Hulk and F. Rempt, "Optokinetic sensations evoked by local stimulation of the peripheral retina," Doc. Ophthalmol. 56(3), 237-242 (1984).

7. M. P. To et al., "Vision out of the corner of the eye," Vision Res. 51(1), 203-214 (2011).

8. R. W. Thatcher, D. North, and C. Biver, "Quantitative EEG and the Frye and Daubert standards of admissibility," Clin. EEG Neurosci. 34(2), 39-53 (2003).

9. R. W. Thatcher, D. North, and C. Biver, "Evaluation and validity of a LORETA normative EEG database," Clin. EEG Neurosci. 36(2), 116-122 (2005).

10. D. Lehman, H. P. Meles, and Z. Mir, "Average multichannel EEG potential fields evoked from upper and lower hemi-retina: latency differences," Electroencephalogr. Clin. Neurophysiol. 43(5), 725-731 (1977).

11. V. Morell, "A 24-hour circadian clock is found in the mammalian retina," Science 272(5260), 349 (1996).

12. K. F. Storch et al., "Intrinsic circadian clock of the mammalian retina: importance for retinal processing of visual information," Cell 130(4), 730-741 (2007).
13. G. Tosini et al., "The circadian clock system in the mammalian retina," Bioessays 30(7), 624-633 (2008).

14. D. S. Gregerson, "Immune privilege in the retina," Ocul. Immunol. Inflammation 6(4), 257-267 (1998).

15. B. Detrick and J. J. Hooks, "Immune regulation in the retina," Immunol. Res. 47(1-3), 153-161 (2010).

16. F. A. Medeiros et al., "Retinal ganglion cell count estimates associated with early development of visual field defects in glaucoma," Ophthalmology 120(4), 736-744 (2013).

17. D. Zelinsky, "Neuro-optometric diagnosis, treatment and rehabilitation following traumatic brain injuries: a brief overview," Phys. Med. Rehabil. Clin. N. Am. 18(1), 87-107 (2007).

18. S. Cochin et al., "Perception of motion and qEEG activity in human adults," Electroencephalogr. Clin. Neurophysiol. 107(4), 287-295 (1998).

19. M. Gaetz and H. Weinberg, "Electrophysiological indices of persistent post-concussion symptoms," Brain Inj. 14(9), 815-832 (2000).

20. U. Samadani et al., "Eye tracking detects disconjugate eye movements associated with structural traumatic brain injury and concussion," J. Neurotrauma 32(8), 548-556 (2015).

21. R. W. Thatcher, "Validity and reliability of quantitative electroencephalography (qEEG)," J. Neurother. 14, 122-152 (2010).

22. J. Duff, "The usefulness of quantitative EEG (QEEG) and neurotherapy in the assessment and treatment of post-concussion syndrome," Clin. EEG. Neurosci. 35(4), 198-209 (2004).

23. M. H. Heitger et al., "Impaired eye movements in post-concussion syndrome indicate suboptimal brain function beyond the influence of depression, malingering or intellectual ability," Brain 132(Pt 10), 2850-2870 (2009).

Deborah Zelinsky is an optometrist whose cutting-edge research in retinal circuitry and neuro-optometric rehabilitation in brain injury has been published and taught worldwide. She founded the Mind-Eye Connection in Northbrook, Illinois, in 1993 and has three patents with novel usages of retinal stimulation. Emphasis is on the development of visual processing in patients needing assessment of brain function-those with injury, neurodegenerative disorders, and/or developmental delays. Treatment involves therapeutic lenses and collaboration with other practitioners.

Corey Feinberg specializes in quantitative EEG assessment and neurofeedback. He has experience in treating a multitude of disorders ranging from anxiety, depression, and attention deficits, to stroke and traumatic brain injuries. He has influenced the field by providing private consultation and original advanced training techniques for numerous leading practitioners nationwide. His strong belief in a collaborative approach has led Corey to working with other local professionals with specialties in neurology, biochemistry, and neuro-optometry. 